\title{
Analyse quantitative du développement végétatif du système aérien de jeunes pêchers (Prunus persica L Batsch)
}

\author{
L Pagès 1, M Génard 1, J Kervella 2 \\ 1 INRA, centre d'Avignon, laboratoire d'agronomie; \\ 2 Station de recherches fruitières méditerranéennes, Domaine St Paul, 84143 Montfavet Cedex, France
}

(Reçu le 20 juillet 1992; accepté le 4 décembre 1992)

\begin{abstract}
Résumé - La croissance et la ramification de jeunes pêchers (Prunus persica $L$ Batsch, cultivars Flavorcrest et Royal Glory) pendant leur première saison de végétation ont été décrites de façon quantitative, à 2 niveaux : le niveau de l'axe et le niveau de métamère (défini comme l'ensemble entre-nœud, nœud, feuille et méristème axillaire). L'étude des courbes de croissance des axes montre que leur allongement, bien que continu tout au long de la saison, présente des phases de ralentissement indépendantes des fluctuations de température. Ces phases de ralentissement apparaissent synchrones au niveau d'un arbre et entre arbres. Au contraire, le nombre de feuilles de chaque axe augmente de façon pratiquement linéaire tout au long de la saison, suivant une vitesse qui dépend de l'ordre de l'axe. L'étude de la croissance et de la ramification au niveau du métamère permet de préciser comment ces 2 processus sont liés. Plus forte est la croissance de l'axe au moment où se développe le métamère, plus son entre-nœud et sa feuille sont longs, et plus s'accroît la probabilité qu'il donne naissance à un axe fils. Pour les axes d'ordre 1, l'axe fils sera le plus souvent un rameau court portant 1-5 feuilles si la vitesse de croissance est intermédiaire et un rameau long si la vitesse est plus forte. Pour un axe d'ordre 2, la probabilité de ne pas observer de ramification est plus élevée que pour un axe d'ordre 1, à vitesse égale de croissance de l'axe porteur. Cette probabilité est plus forte quand la feuille axillante est en-dessous de l'axe porteur, et plus faible quand elle est au-dessus. Les courbes d'évolution des probabilités de production des axes longs ou courts en relation avec les vitesses de croissance des axes porteurs sont présentées. II semble que la variété influence également ces probabilités. La croissance des rameaux axillaires n'est dépendante que de manière fugace du contexte de formation du métamère porteur.
\end{abstract}

Prunus persica $=$ pêcher $/$ croissance $/$ ramification $/$ développement $/$ analyse quantitative

Summary - Quantitative analysis of the vegetative development of the shoot system of young peach trees. The growth and branching processes occurring during the first growing season of Flavorcrest and Royal Glory peach (Prunus persica $L$ Batsch) trees have been described quantitatively. The axis level and the metamer (set of internode, node, leaf and associated bud) level were considered. Axes elongated continuously throughout the growing season, but with growth rate variations (figs $2 A, B$ ). These variations tended to be synchronous within a tree and between trees. On the contrary, the number of leaves at each axis increased at a constant rate (figs $2 C, D$ ). This rate depended on the branching order of the axis. The study of growth and branching at the metamer level showed how these 2 processes were related. Leaves and internodes of metamers tended to be longer if the axis they composed grew fast while they were being formed. Their axillary bud was also less likely to remain dormant in such a case. On first-order axes, buds grew most frequently into a short branch with 1-5 leaves if the growth rate of the axis was intermediate, and into a long shoot if the growth rate was high (fig 4). On second-order axes, the probability for a bud to grow into a shoot was lower and was significantly influenced by the position of the leaf around the axis (fig 5). The later growth of an axillary shoot was only slightly influenced by the growth rate of the father axis during the formation of the metamer bearing this shoot.

Prunus persica $=$ peach $/$ tree $/$ growth $/$ branching $/$ development $/$ quantitative analysis 


\section{INTRODUCTION}

Comme le montrent les travaux concernant le pommier, une recherche conjointe de formes nouvelles et de cultivars adaptés à ces formes (Lespinasse et Delort, 1990; Quinlan et Tobutt, 1990) permet d'améliorer la rentabilité des cultures. Elle mérite donc d'être entreprise pour d'autres espèces d'arbres fruitiers et plus particulièrement pour le pêcher, deuxième espèce fruitière en France et dont l'importance est particulièrement grande dans le Sud-Est. Une telle recherche repose sur une bonne connaissance du développement végétatif (organogénèse et croissance) de l'arbre (Lespinasse et Delort, 1988; Harris, 1990). Nous nous proposons donc dans un premier temps d'étudier le développement végétatif du pêcher. L'objectif de cette étude est d'acquérir les connaissances et de définir les hypothèses nécessaires à une future modélisation mathématique du développement utilisable pour tester par simulation l'effet de nouvaux modes de conduite.

Des descriptions qualitatives ou semiquantitatives dynamiques du développement du pêcher ont déjà été réalisées (Champagnat, 1954; Champagnat, 1965; Monet, 1983; Lauri, 1991). Ces travaux ont notamment mis en évidence l'existence de liaisons entre les processus de croissance et de ramification d'un rameau donné. Plus récemment la variabilité génétique du développement a été étudiée quantitativement (Scorza, 1984; Scorza, 1987; Scorza et al, 1989; Kervella, 1991). Cependant, ces recherches portent sur la comparaison de la morphologie de différentes variétés à un âge donné et n'abordent pas l'étude dynamique du développement du pêcher.

Cet article récapitule la première étape d'une étude quantitative et dynamique des processus de croissance et de ramification sur 2 variétés. Nous présentons les résultats obtenus pendant la première année de végétation, au cours de laquelle la forme du futur arbre commence à se mettre en place. Après avoir décrit le développement à l'échelle de l'arbre entier et du rameau, nous étudions le développement au niveau du métamère, défini comme l'ensemble : nœud, entre-nœud, feuille, méristème axillaire (White, 1979; Barlow, 1992). Le métamère a été choisi comme unité de travail car il peut être considéré comme l'unité de base de construction de l'arbre. La croissance des axes d'un arbre résulte en effet de la production de nouveaux mé- tamères et de leur allongement, et leur ramification de l'apparition d'axes fils sur les métamères. La croissance ainsi que les relations entre croissance et ramification seront étudiées grâce à une approche multivariée (Jeune et Sattler, 1992) en analysant les liaisons entre le contexte de la formation d'un métamère, ses caractéristiques finales et le devenir de son méristème axillaire.

\section{MATÉRIEL ET MÉTHODES}

\section{Matériel et culture}

Pour réaliser cette étude, nous avons utilisé les variétés de pêcher Flavorcrest et Royal Glory greffées sur de jeunes semis du porte-greffe INRA GF 305.

Les jeunes pêchers ont été installés en février 1990 en conteneurs de 80 | placés en plein air et protégés du vent par un filet semi-perméable. Ils ont été greffés au début de mai.

Pendant toute la culture, les arbres ont été irrigués et fertilisés grâce à une solution nutritive obtenue par dilution d'un engrais soluble (solution N-P-K dans les proportions 12-9-34, avec oligo-éléments) et distribuée à l'aide d'un système de goutte à goutte (jusqu'à faible drainage).

Leur état phytosanitaire a été surveillé 2 fois par semaine. Nous avons ainsi évité toute attaque parasitaire importante.

\section{Observations et mesures}

Elles ont été réalisées sur 15 arbres (8 de la variété Flavorcrest, et 7 de la variété Royal Glory), 2-3 fois par semaine de mai à août, et 1 fois par semaine en septembre.

La croissance des axes a été apparemment continue durant la saison de végétation. Pendant toute cette période, à l'extrémité de chaque axe, 6-8 jeunes feuilles non déployées (dont les 2 parties du limbe ne sont pas totalement décollées l'une de l'autre) étaient visible au-dessus de la dernière feuille déployée. L'apparition de rameaux axillaires était presque concomitante de la formation de la feuille axillante : les premières feuilles des rameaux axillaires étaient déjà visibles à l'aisselle des feuilles non déployées.

La croissance a été suivie en mesurant pour chacun des axes sa longueur totale, de sa base jusqu'à l'extrémité de son apex, et en comptant le nombre de feuilles déployées. La longueur du limbe de chacune de ces feuilles a également été mesurée.

La ramification a été décrite en notant à chaque fois les nouveaux axes apparus, identifiés par la connais- 
sance de leur axe père et du rang de la feuille axillante sur cet axe.

Les températures minimales et maximales quotidiennes ont été mesurées sous abri à proximité du site de culture.

\section{Analyses}

Pour pouvoir étudier et comparer les cinétiques de développement, obtenues en conditions de températures variables, nous nous sommes ramenés à une échelle du temps plus «biologique» en utilisant le modèle suivant :

$$
\text { Temps }=\sum_{i=1}^{n}\left[\min \left(T_{o p t}, t_{i}\right)-T_{\text {seuil }}\right]
$$

où Temps est la nouvelle échelle de temps (en degrés.jours); $n$ le nombre de jours écoulés à la date considérée depuis la date de greffage; $\min \left(T_{o p t}, t_{i}\right)$ la valeur la plus faible entre $T_{\text {opt }}$ la température optimale de développement, et $t_{j}$ la température moyenne du jour $i ; T_{\text {seuil }}$ la température seuil en déçà de laquelle on n'observe pas de développement. La valeur de $\mathrm{T}_{\text {opt }}$ a été fixée à $30{ }^{\circ} \mathrm{C}$ (optimum pour la photosynthèse selon Anon, 1987) et celle de $T_{\text {seuil }}$ à $3{ }^{\circ} \mathrm{C}$ (en l'absence de données sur un seuil de croissance végétative, nous avons pris la valeur obtenue sur ébauches florales par Rageau, 1982).

On suppose donc que la réponse de la vitesse de développement avec la température est linéaire, pour des températures variant de $T_{\text {seuil }}$ à $T_{\text {opt }}$.

Les surfaces foliaires ont été estimées d'après les longueurs de feuilles mesurées, en utilisant une relation établie (étalonnée) à partir des mesures de longueur et surface de 198 feuilles prélevées sur des arbres cultivés dans les mêmes conditions mais non étudiés. Cette relation :

$$
\text { surface }=\text { a. } \text { longueur }{ }^{b}
$$

avec $a=0,453$ et $b=1,76$ expliquait plus de $95 \%$ de la variance totale des surfaces foliaires de l'ensemble des feuilles, prélevées à différentes dates, sur des axes d'ordre 1,2 et 3 et sur les 2 variétés considérées.

Le développement de l'arbre entier a été décrit par des représentations graphiques de la distribution des axes d'ordre 2 le long du tronc et par les courbes de croissance en longueur et en nombre de métamères formés (considéré comme égal au nombre de feuilles déployées) des axes des principaux ordres. Le caractère non aléatoire de la distribution des ramifications le long de l'axe d'ordre 1 a été testé par un test de «runs» (Siegel, 1956).

Le développement de 633 métamères appartenant à des axes d'ordre 1 et de 6049 métamères appartenant à des axes d'ordre 2 a été décrit. Pour cela, chaque métamère a été caractérisé par des variables dé- crivant le contexte de son apparition, ses caractéristiques finales, et des variables décrivant la croissance de l'axe fils éventuel, ainsi que par son origine variétale. Pour décrire le contexte d'apparition d'un métamère, nous avons pris en compte des caractéristiques de croissance de l'axe auquel il appartient (que nous appellerons "axe porteur") au cours des 120 degrés.jours (approximativement 6 j) précédant la date de déploiement de la feuille du métamère considéré, c'est-à-dire pendant la formation de ce métamère. Nous avons calculé les vitesses moyennes (accroissement/durée) de croissance en longueur ( $v l)$, en nombre de métamères ( $v n$ ) et en surface foliaire (vs) de cet axe porteur ainsi que la longueur moyenne des entre-nceuds qu'il a produits (le) sur cet intervalle de temps. Les accroissements des variables considérées au cours de ces 120 degrés.jours ont été calculés après interpolation linéaire des données aux dates souhaitées. Pour les axes d'ordre 2, la croissance au niveau de l'arbre a également été caractérisée, par le calcul de la vitesse de croissance en longueur cumulée des axes d'ordre 2 (VLT).

L'état final du métamère a été caractérisé par la longueur de la feuille (If) et le type de ramification à son aisselle. Des variables indicatrices ont été définies pour les différents types de ramifications distingués : métamères sans axe fils (variable indicatrice $F_{0}$ ), métamères avec axes courts (portant de 1 à 5 feuilles: variable indicatrice $F_{1}$ ) et métamères avec axes longs (axes allongés portant plus de 5 feuilles: variable indicatrice $F_{2}$ ). Pour les métamères d'ordre 2 sans ramification, une distinction a également été établie suivant que leur feuille est située au-dessus $\left(F_{01}\right)$ ou au-dessous du rameau $\left(F_{02}\right)$.

Pour décrire la croissance de l'axe fils d'un métamère, nous avons retenu ses accroissements en nombre de métamères sur 4 périodes de 200 degrés.jours successives à partir de la date de déploiement de la feuille (respectivement $n_{1}, n_{2}, n_{3}, n_{4}$ ). De ce fait, les métamères apparus moins de 800 degrés.jours avant l'arrêt de la végétation, c'est-à-dire après le 21 août, n'ont pas été pris en compte dans l'étude. Ces accroissements ont également été calculés par interpolation linéaire des données aux dates souhaitées.

Deux variables indicatrices de la variété d'origine ( $F$ pour Flavorcrest et $R$ pour Royal Glory) ont également été définies.

Les variables utilisées sont récapitulées dans les tableaux I et II.

La variabilité du développement des métamères est étudiée grâce à des analyses en composantes principales (ACP) normées (Lebart et al, 1984), réalisées séparément pour les métamères appartenant à des axes d'ordre 1 et pour les métamères appartenant à des axes d'ordre 2 , décrits par les variables présentées ci-dessus. Les variables décrivant qualitativement le devenir du métamère, la variété et la croissance en longueur de l'ensemble des axes d'ordre 2 de l'arbre (tableaux I et II) sont intervenues comme variables inactives (appelées aussi variables 
Tableau I. Liste des variables utilisées dans I'ACP sur les métamères d'ordre 1.

Variables Symbole Unité

Variables principales (actives)

\section{Axe père}

Vitesse de croissance en surface foliaire

Vitesse de croissance en nombre de métamères

Vitesse de croissance en longueur

Longueur moyenne des entrenœuds

\section{Métamère}

Longueur atteinte par la feuille axillante

Axes fils

${ }^{*}$ Métamères ${ }_{0-200}$

* Métamères $200-400$

* Métamères $400-600$

* Métamères $600-800$

Variables supplémentaires (inactives)

Type de ramification

Métamère sans axe fils

Métamère avec axe fils court ( $1-5$ feuilles)

Métamère avec axe fils long

Variété

Flavorcrest

Royal Glory

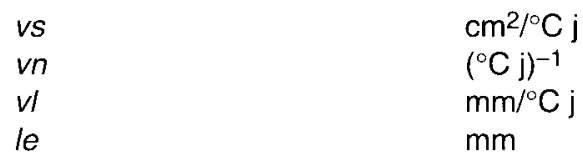

If $\quad \mathrm{mm}$

$\begin{array}{ll}n_{1} & \cdots \\ n_{2} & \cdots \\ n_{3} & \cdots \\ n_{4} & \cdots\end{array}$

${ }^{*}$ Métamères $x_{x-y}$ : nombre de métamères formés entre $x$ et $y^{\circ} \mathrm{C} j$ après la formation du métamère père.

supplémentaires) dans l'analyse. Cette procédure permet de mettre en évidence d'éventuelles liaisons entre ces variables et celles décrivant le développement du métamère.

Les variables sont représentées par des vecteurs normés que l'on projette sur les plans factoriels. La proximité de la projection de la variable au cercle des corrélations (cercle de rayon 1, la valeur 1 étant la distance maximale à l'origine de la projection de la variable) donne la qualité de la représentation dans le plan étudié. Le cosinus de l'angle entre les projections de 2 variables (ou d'une variable et d'un axe) mesure la corrélation entre ces 2 variables (ou entre la variable et l'axe) dans le plan. Quand 2 variables sont à la fois bien représentées et ont un angle fermé entre elles dans le plan, c'est qu'elles sont corrélées dans l'absolu.

Pour préciser la liaison entre la vigueur d'un axe et sa ramification, nous avons étudié dans nos populations de métamères la variation de la proportion de ceux sans axe fils selon la vitesse d'allongement de l'axe père. Concernant les axes d'ordre 1 , nous avons en outre calculé la proportion de métamères portant un axe court et la proportion de ceux portant un axe long. Pour les axes d'ordre 2 la variation de la proportion de métamères sans axe fils avec la vigueur de l'axe porteur a été étudiée séparément pour les métamères dont la feuille est située sous et au-dessus de l'axe. Ces proportions sont obtenues par le calcul de moyennes glissantes des variables $F_{0}, F_{01}, F_{02}, F_{1}$ et $F_{2}$ (tableaux I et II) le long de l'axe des vitesses (variable vl). Nous avons utilisé l'algorithme "ksmooth" décrit par Chambers et al, 1983.

\section{RÉSULTATS}

\section{Développement de l'arbre}

Pendant cette première saison de végétation, les arbres se sont ramifiés jusqu'à l'ordre 3 à partir 
Tableau II. Liste des variables utilisées dans l'ACP sur les métamères d'ordre 2.

Variables Symbole Unité

Variables principales (actives)

\section{Axe père}

Vitesse de croissance en surface foliaire

Vitesse de croissance en nombre de métamères

Vitesse de croissance en longueur

Longueur moyenne des entrenœuds

$\begin{array}{ll}\text { vs } & \mathrm{cm}^{2 /}{ }^{\circ} \mathrm{C} \mathrm{j} \\ \text { vn } & \left({ }^{\circ} \mathrm{C} \mathrm{j}\right)^{-1} \\ v l & \mathrm{~mm} /{ }^{\circ} \mathrm{C} \mathrm{j} \\ l e & \mathrm{~mm}\end{array}$

\section{Métamère}

Longueur atteinte par la feuille axillante

$\mathrm{mm}$

Axes fils

* Métamères $0-200$

* Métamères $200-400$

* Métamères $400-600$

* Métamères $600-800$

$\begin{array}{ll}n_{1} & -- \\ n_{2} & -- \\ n_{3} & \cdots \\ n_{4} & \cdots\end{array}$

Variables supplémentaires (inactives)

Métamère sans axe fils

Métamère sans axe fils (feuille sous l'axe)

Métamère sans axe fils (feuille au-dessus de l'axe)

Métamère avec axe fils court ( $1-5$ feuilles)

Métamère avec axe fils long

Variété Flavorcrest

Variété Royal Glory

Vitesse de croissance en longueur de l'ensemble des axes 2

$\begin{array}{ll}F_{0} & 1 \text { ou } 0 \\ F_{01} & 1 \text { ou } 0 \\ F_{02} & 1 \text { ou } 0 \\ F_{1} & 1 \text { ou } 0 \\ F_{2} & 1 \text { ou } 0 \\ F & \\ R & 1 \text { ou } 0 \\ \text { VLT } & 1 \text { ou } 0 \\ & \mathrm{~mm} /{ }^{\circ} \mathrm{C} \mathrm{j}\end{array}$

* Métamères $s_{x-y}$ : nombre de métamères formés entre $x$ et $y^{\circ} \mathrm{C} j$ après la formation du métamère père.

des bourgeons greffés. Les axes d'ordre 2 et 3 que nous avons observés sont des axes sylleptiques. Les axes obtenus étaient tous plus ou moins orthotropes. Les différences entre les axes sont surtout des différences quantitatives, de croissance, longueurs d'entre-nœuds, longueurs des feuilles, densité de ramification.

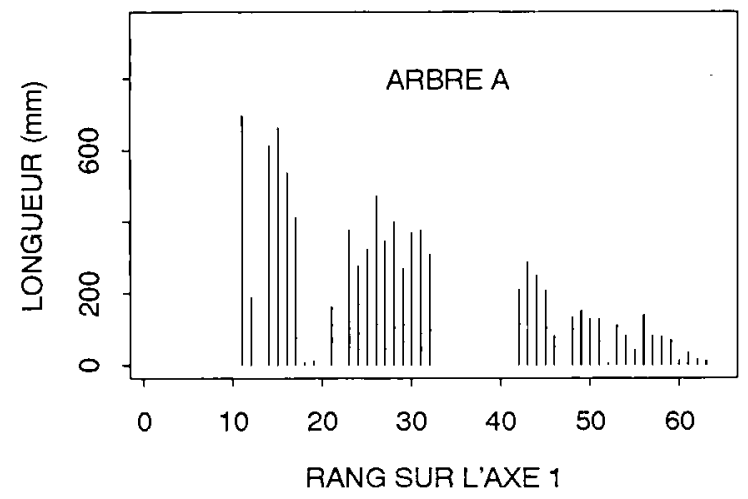

La production des rameaux anticipés le long des axes d'ordre 1 et 2 s'est produite par vagues plus ou moins nettes suivant les axes. La figure 1 illustre ce point sur 2 arbres. Le caractère agrégé de la répartition des axes fils le long des axes d'ordre 1 a été confirmé pour chaque variété par le test de “runs» réalisé sur chacun des arbres.

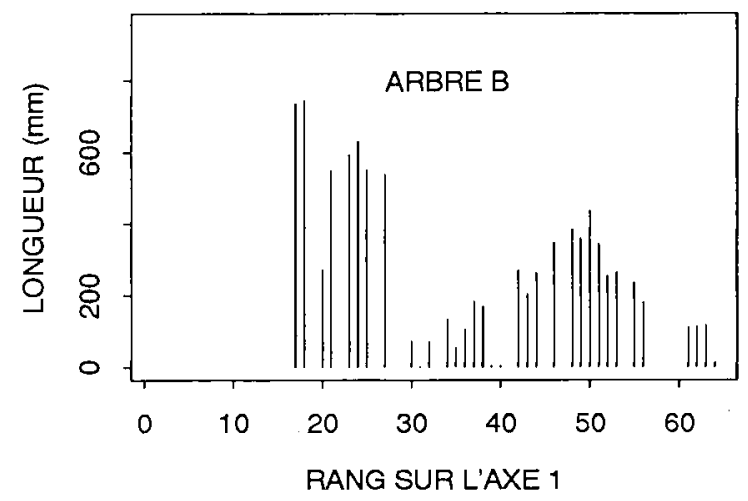

Fig 1. Niveau d'apparition et longueur des axes d'ordre 2 le long des axes d'ordre 1 en fin de saison de croissance : exemple de 2 arbres. 
Les courbes de croissance en longueur des axes sont à 1 phase (sigmoïde simple), à 2 phases (double sigmoïde), voire à 3 phases (triple sigmoïde) sur certains axes d'ordre 2. Sur la figure $2 A, B$, nous présentons des exemples de ces courbes pour 2 arbres. Les phases d'accélération ou, au contraire, de décélération de croissance sont souvent synchrones, à la fois entre les différents axes d'un même arbre et entre arbres (ce dernier point a été mis en évidence grâce à l'observation d'un grand nombre de courbes de croissance que nous ne
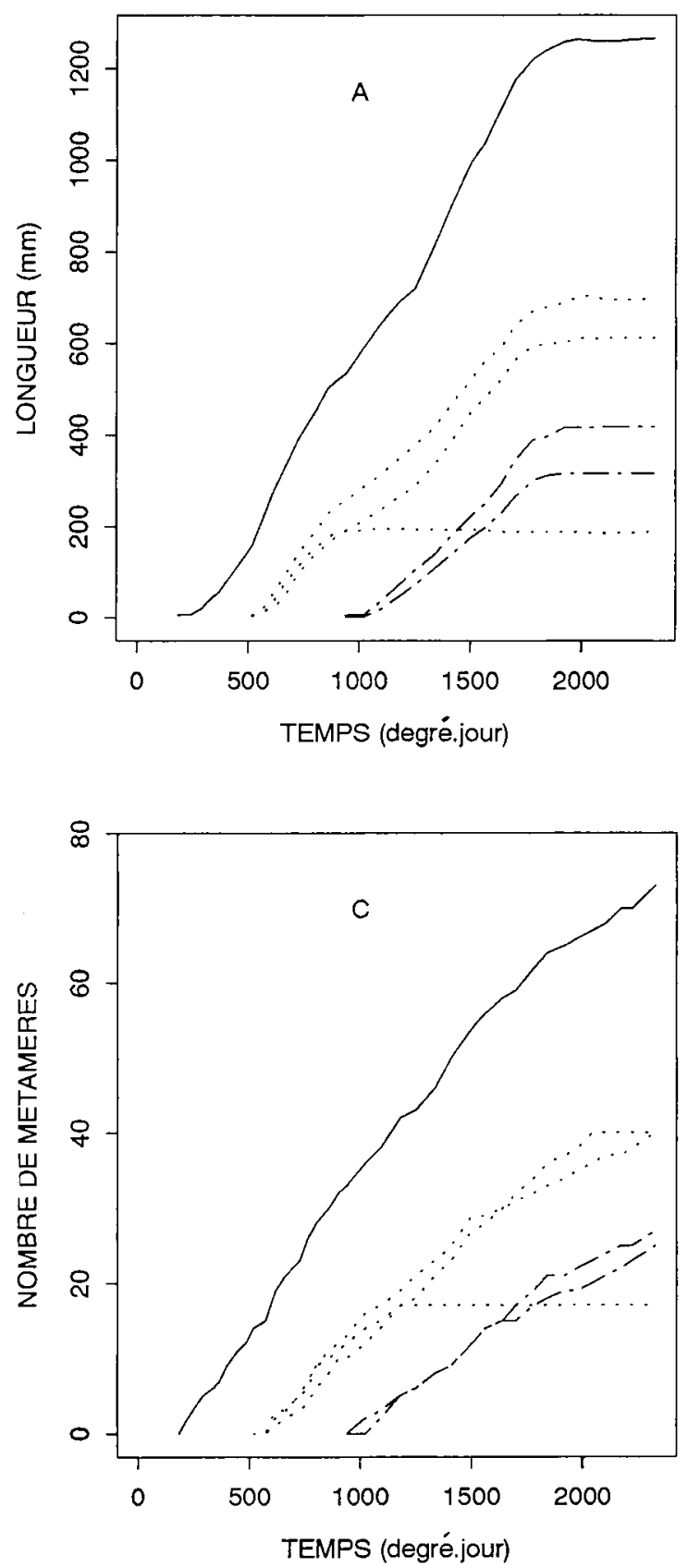

pouvons présenter ici). Au contraire des courbes de croissance en longueur, les courbes de croissance en nombre de métamères (fig $2 \mathrm{C}, \mathrm{D}$ ) sont pratiquement linéaires, avec toutefois une légère tendance à la diminution de la vitesse au cours du temps. La pente générale de ces courbes est plus forte pour les axes d'ordre 1 que pour les autres axes. Le plastochrone apparent des axes d'ordre 1 est donc le plus faible. Nous n'avons pas observé de différences entre les courbes de croissance provenant des 2 variétés.
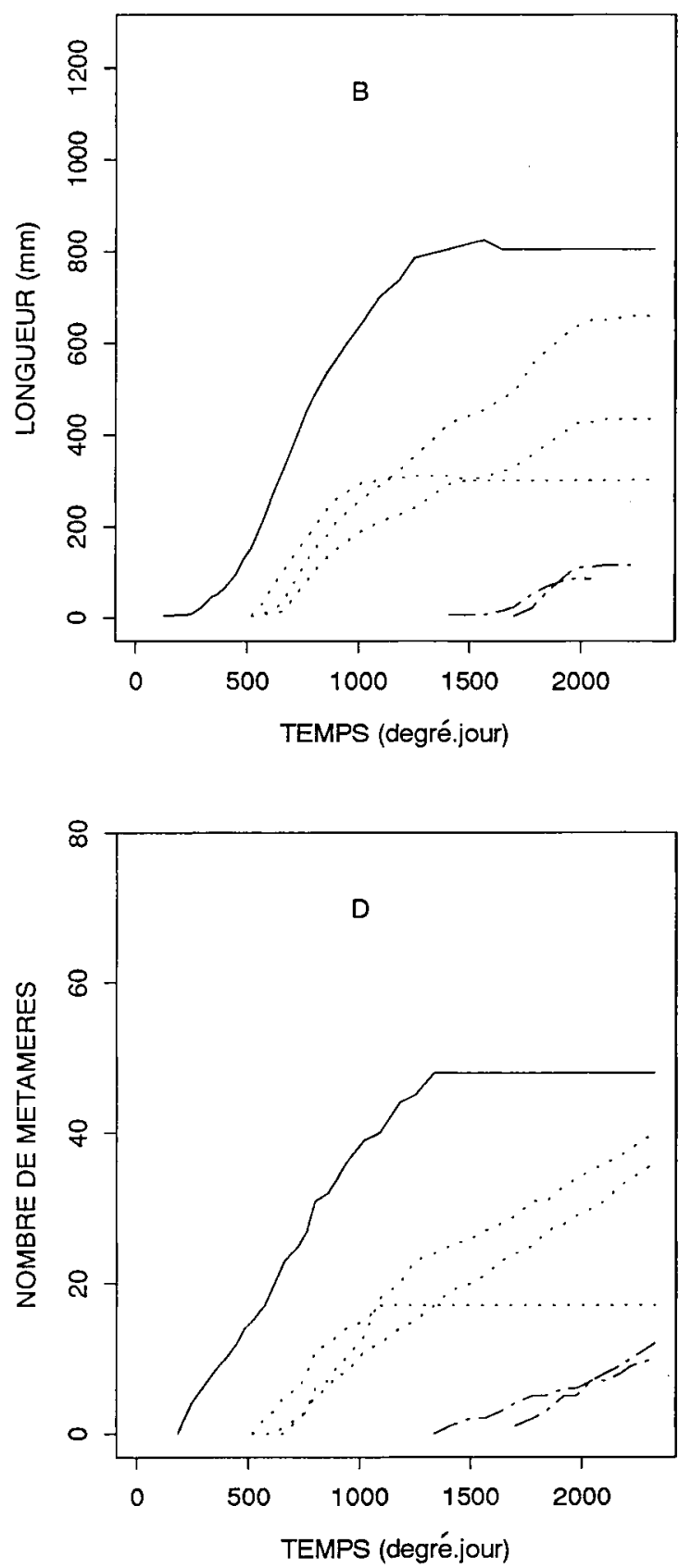

Fig 2. Courbes de croissance en longueur ( $A$ et $B$ ) et en nombre de métamères (C et $D$ ) pour des axes d'ordre 1 (traits pleins), d'ordre 2 (traits pointillés), et d'ordre 3 (tiretés). Les graphiques $A$ et $C$ présentent les courbes types des mêmes 6 axes pris sur un seul arbre. De même, les graphiques $B$ et $D$ sont les courbes des mêmes 6 axes sur un autre arbre. 


\section{Développement des métamères}

Dans l'interprétation des ACP sur les métamères, nous n'avons retenu que le premier plan factoriel, qui extrait $70 \%$ de l'inertie dans le cas des métamères d'ordre 1 et $60 \%$ dans celui des métamères d'ordre 2. À une légère rotation près, les variables se projettent de façon similaire sur ce plan dans les 2 cas (fig 3). Elles forment 2 groupes distincts : le premier réunit les variables décrivant le contexte de formation du métamère (vitesses de croissance de l'axe porteur et, pour les axes d'ordre 2, de l'ensemble des axes) ainsi que la longueur de sa feuille; le second regroupe les variables concernant la croissance de l'axe fils.

Ces 2 groupes ont des positions voisines le long du premier axe factoriel, que l'on peut interpréter comme décrivant la "vigueur générale» du métamère. Les variables indicatrices du type de ramification $\left(F_{0}, F_{1}\right.$ et $\left.F_{2}\right)$ s'échelonnent le long de ce premier axe. Ces variables, inactives dans I'ACP, illustrent l'augmentation de l'intensité de ramification d'un métamère avec sa vigueur.

Les courbes représentant les proportions de métamères avec ou sans ramification en fonction de la vitesse de croissance en longueur de l'axe porteur ( $(\mathrm{l})$, descripteur classique de la vigueur, précisent cette liaison entre vigueur et ramification. Ainsi, pour les axes d'ordre 1 (fig 4), près de $60 \%$ des métamères n'ont pas d'axe fils pour des vitesses inférieures à $0,2 \mathrm{~mm} /$ degré-jour. Pour des vitesses comprises entre 0,2 et $0,4 \mathrm{~mm} /$ degrés-jour (soit environ 4-8 $\mathrm{mm} /$ jour) les ramifications qui apparaissent sont surtout des axes courts. La fréquence d'axes longs devient élevée lorsque la vitesse de croissance est $>0.4$ $\mathrm{mm} /$ degré-jour (ce qui correspond approximativement à une vitesse de $8 \mathrm{~mm} / \mathrm{j}$ ). Pour les axes d'ordre 2 (fig 5), les formes de ces courbes sont voisines de celles observées pour les axes d'ordre 1 , avec une proportion plus forte de métamères sans axe fils pour une vitesse de croissance donnée. La différence entre les métamères dont la feuille est située au-dessus du rameau et ceux dont la feuille est située audessous est notable, la ramification étant plus fréquente sous le rameau. En observant les courbes obtenues pour chacun des arbres pris individuellement (résultats non présentés), nous avons pu observer la stabilité de la forme de ces courbes. Les formes des courbes sont très proches pour les 2 variétés, avec de légères différences quantitatives. La variété Flavorcrest semble plus ramifiée, elle produit notamment une plus grande proportion d'axes longs sur l'axe d'ordre 1 (fig 4).

Les variables du second groupe relatives à la ramification $\left(n_{1}, n_{2}, n_{3}\right.$ et $\left.n_{4}\right)$ sont ordonnées le long du deuxième axe. Elles sont de plus en plus corrélées entre elles et de moins en moins avec les variables du premier groupe. On note une légère différence entre l'ordre 2 , où les va-

\section{METAMERES D'ORDRE 1}

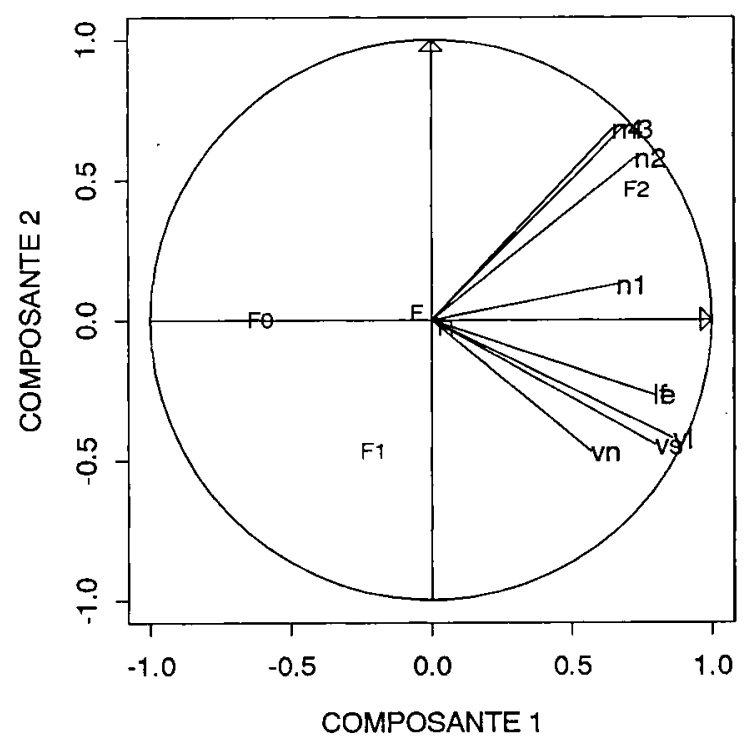

\section{METAMERES D'ORDRE 2}

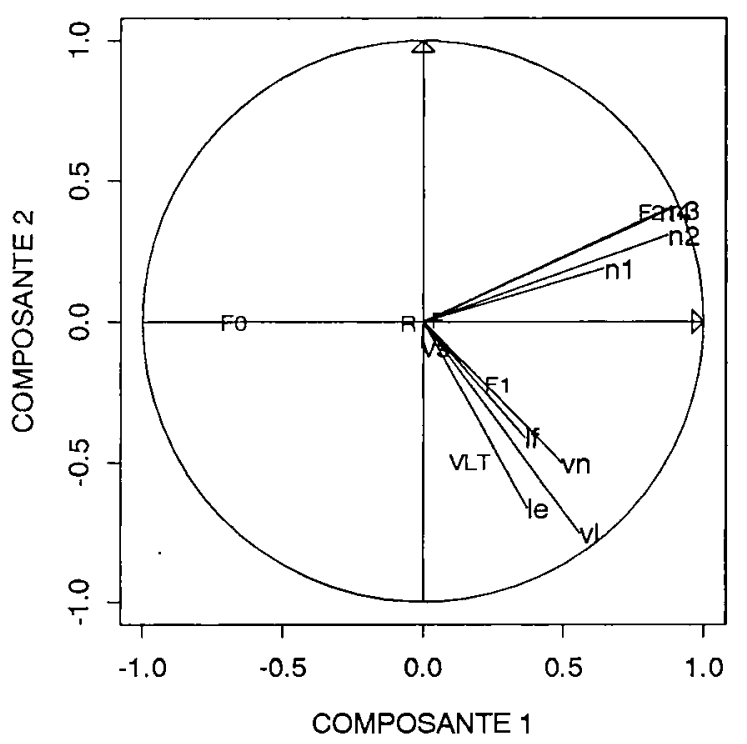

Fig 3. Projection des variables sur le premier plan factoriel pour les ACP réalisées avec les variables relatives aux métamères d'ordre 1 et d'ordre 2 répertoriées aux tableaux I et II. Les variables principales, en lettres minuscules, sont représentées par des vecteurs, les variables supplémentaires apparaissent en lettres majuscules. 

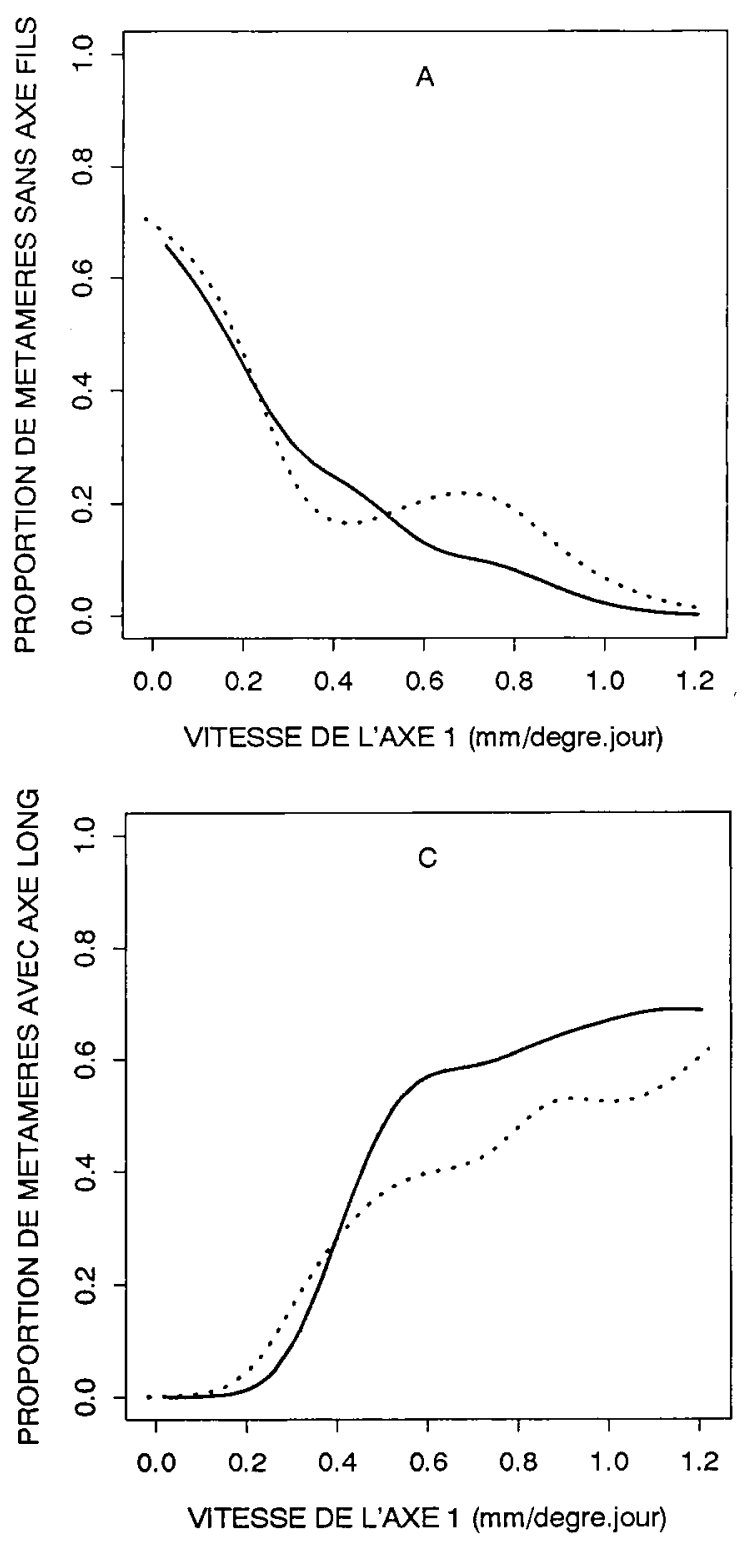

riables du second groupe sont groupées, et l'ordre 1 , où la variable $n 1$ se détache plus nettement pour se rapprocher des variables du premier groupe.

Les variables supplémentaires codant pour les variétés sont très mal représentées dans ce premier plan factoriel. Une ACP réalisée sur chacune des variétés a d'ailleurs montré des résultats identiques (résultats non présentés).

\section{DISCUSSION}

Notre étude confirme et permet de préciser la liaison existant entre croissance et ramification sylleptique chez le pêcher, relation mise en évidence par Champagnat (1954) et observée également par Crabbé (1987). Une croissance en

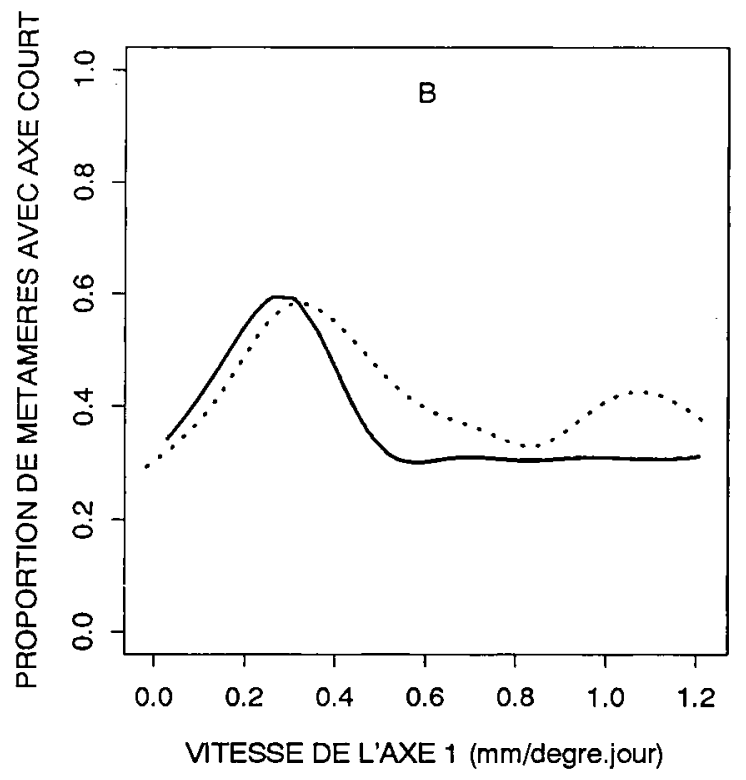

Fig 4. (A) Relation entre la vitesse de croissance de l'axe d'ordre $1(\mathrm{Vl})$ et la proportion de métamères sans axe fils. (B) Relation entre la vitesse de croissance de l'axe d'ordre 1 (VI) et la proportion de métamère portant des axes courts. (C) Relation entre la vitesse de croissance de l'axe d'ordre 1 (vi) et la proportion de métamères portant des axes longs. La variété Flavorcrest est représentée en traits pleins, la variété Royal Glory en traits pointillés.

longueur rapide favorise la ramification sylleptique. La nature du rameau fils éventuel (rameau court ou rameau long) est également liée à la vitesse de croissance en longueur. Les vitesses de croissance seuils données par Champagnat (1954) pour le pêcher, au-dessus desquelles l'axe d'ordre 1 se ramifie et donne un axe court ou un axe long sont respectivement $5,5 \mathrm{~mm}$ et $14,5 \mathrm{~mm} / \mathrm{j}$. Elles sont du même ordre de grandeur que celles que nous trouvons ( 4 et $8 \mathrm{~mm} / \mathrm{j}$ ). Cependant, contrairement aux observations de Champagnat (1954), nous n'avons pas observé de seuil en deçà duquel il n'y aurait aucune ramification. Nous avons plutôt constaté que la ramification évoluait gradueliement en relation avec la vitesse de croissance de l'axe porteur. Vu l'absence d'un déterminisme à seuil, nous avons été conduits à considérer des probabilités de ramification. Ces probabilités dépendent dans notre 


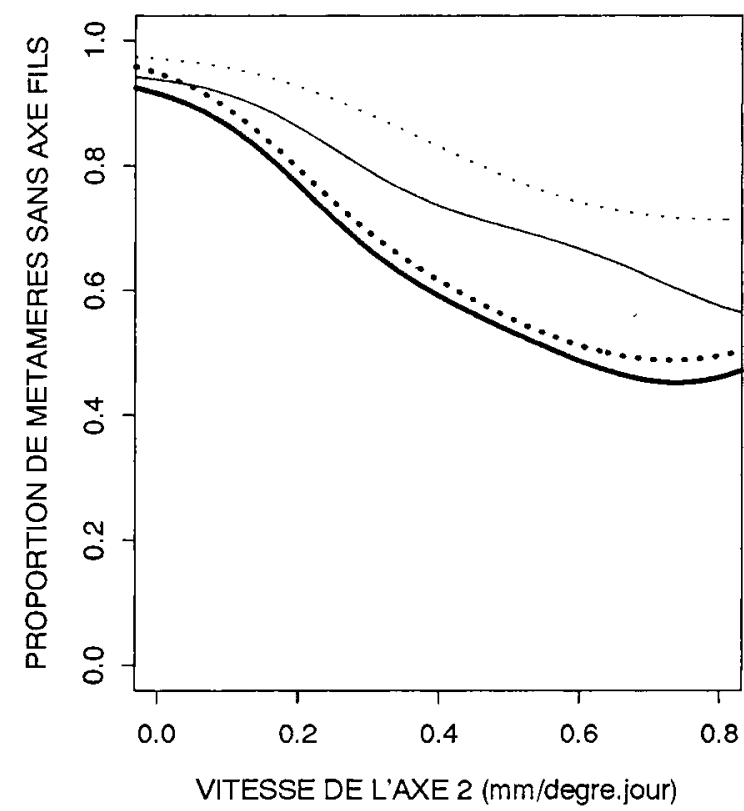

Fig 5. Relation entre la vitesse de croissance de l'axe d'ordre 2 ( $V l)$ et la proportion de métamères sans axe fils, parmi les métamères dont les feuilles sont situées en dessous (traits épais) et au-dessus (traits fins) du rameau. La variété Flavorcrest est représentée en traits pleins, la variété Royal Glory en traits pointillés.

cas de l'ordre de ramification considéré : les axes d'ordre 1 ont une plus forte propension à se ramifier. Pour les axes d'ordre 2, qui sont inclinés, elles dépendent également de la position de l'aisselle de la feuille autour du rameau porteur. Les méristèmes axillaires situés au-dessus du rameau se développent plus rarement que ceux situés en-dessous. Ce mécanisme, observé chez de nombreux arbres, et appelé hypotonie (Champagnat, 1947), est très important quant à ses conséquences sur le port de l'arbre, qui aura ainsi tendance à s'étaler. La variété semble également influer sur ces probabilités, Flavorcrest produisant plus fréquemment des rameaux longs sur les axes d'ordre 1 et se ramifiant plus sur les axes d'ordre 2 que Royal Glory. D'après les résultats de Champagnat (1954), ces probabilités pourraient évoluer également au cours de la saison, la ramification devenant de plus en plus rare. Ce point reste à approfondir.

Si la croissance du rameau porteur au moment de la formation du métamère influe sur la nature de la ramification, et sur la croissance initiale du rameau fils, elle influe peu par contre, sur sa croissance ultérieure lorsque ce rameau fils est long. L'effet "vitesse de croissance» du rameau père ne se fait sentir que durant les 200 premiers degrés-jours (environ $10 \mathrm{j}$ ) pour un métamère d'ordre 1 et est pratiquement nul pour un métamère d'ordre 2, comme le montrent les corrélations entre les variables décrivant la vitesse de croissance du rameau fils et celles décrivant la vitesse de croissance du rameau porteur. Ce résultat confirme la vision de White (1979) selon laquelle les axes deviennent de plus en plus indépendants au fur et à mesure que la complexité du système s'accroît.

Le suivi du développement végétatif des pêchers au cours de leur première année nous montre que si la croissance en longueur des rameaux du pêcher s'opère de façon continue dans le temps, elle présente une ou plusieurs phases de ralentissement. Ce type d'observation a également été fait chez le pommier (Crabbé, 1987). Avec la relation entre vitesse de croissance en longueur et ramification, ces fluctuations de la croissance en longueur expliquent la répartition en vagues des rameaux d'ordre 2 le long des axes d'ordre 1. Elles ne sont pas dues à des changements du plastochrone apparent au cours de la croissance, lequel est relativement constant comme cela est souvent observé chez les végétaux ligneux (Crabbé, 1987). II faut donc penser à une diminution de la croissance en longueur de certains entre-nœuds lors des phases de ralentissement. Les causes de ces fluctuations sont encore mal élucidées et pourraient être liées à des régulations internes à l'arbre ou à des variations non maîtrisées des conditions environnementales non prises en compte au travers du "temps biologique" utilisé. L'étude des courbes de croissance en longueur et, pour les axes d'ordre 2, de la liaison entre la croissance en longueur de l'ensemble des axes et celle d'un rameau particulier montrent que, dans nos conditions de culture, les phases de ralentissement sont relativement synchrones, y compris entre arbres. Ceci tendrait à orienter l'interprétation des fluctuations de croissance vers un effet des conditions environnementales. II importe de voir si ce phénomène est plus général, car une relation entre les axes serait à prendre en compte dans une modélisation future.

D'un point de vue méthodologique, l'étude des métamères a montré qu'il est possible d'utiliser certains caractères morphologiques (taille de la feuille, longueur des entre-nœuds, type de ramification) pour retracer a posteriori l'historique de la croissance d'un rameau. II convient toutefois de confirmer plus généralement leur valeur pour comparer ainsi différentes situations de culture ou différents génotypes. Le choix des métamères comme unité d'analyse a permis un début 
de quantification de la propension à la ramification et de l'hypotonie. Nous avons ainsi pu mettre en évidence des différences entre les variétés, Flavorcrest produisant plus fréquemment des rameaux longs sur les axes d'ordre 1 et se ramifiant plus sur les axes d'ordre 2. II conviendra de développer cette approche pour l'utiliser dans l'étude de la variabilité génétique des phénomènes de croissance et de ramification.

Au travers des analyses effectuées, nous avons pu noter l'homogénéité des comportements morphogénétiques entre les ordres de ramification d'une part, et entre les 2 variétés d'autre part. Seules des variations quantitatives (plastochrone différent selon l'ordre, probabilités de ramification différentes selon la variété) ont été notées. Si ceci se confirme, nous pourrons envisager les mêmes règles générales en vue de la modélisation du développement des différents ordres et des différentes variétés.

\section{REMERCIEMENTS}

Nous remercions $G$ Ducailar pour sa collaboration technique lors de l'expérimentation, $T$ Pascal et $\mathrm{J}$ Aymard pour leurs conseils dans le choix du matériel végétal, ainsi que $F$ Lescourret pour ses remarques dans l'élaboration du manuscrit.

\section{RÉFÉRENCES}

Anon (1987) L'arbre et son milieu; exigences climatiques. In : Le pêcher, références et techniques ( $\mathrm{J}$ Vidaud, I Jacoutet, J Thivend, eds) CTIFL, Paris, 74-93

Barlow PW (1992) From cell to system: repetitive unit of growth in the development of shoot and roots. In: Growth patterns in vascular plants (M Iqbal, ed) Dioscorides Press, Portland, Oregon, USA, 25-42

Chambers JM, Cleveland WS, Kleiner B, Tukey PA (1983) Graphical methods for data analysis. Wadsworth International Group, Belmont, California

Champagnat $P$ (1947) Les principes généraux de la ramification des végétaux ligneux. Rev Hortic 2143 , 335-341

Champagnat P (1954) Recherches sur les rameaux anticipés des végétaux ligneux. Rev Cytol Biol Vég $15,1-51$
Champagnat $P$ (1965) Rameaux courts et rameaux longs : problèmes physiologiques. Handb Pflanzenphysiol 15, 1165-1171

Crabbé J (1987) Aspects particuliers de la morphogénèse caulinaire des végétaux ligneux et introduction à leur étude quantitative. IRSIA, Bruxelles, $117 \mathrm{p}$

Harris RW (1990) Tree structure in relation to performance: introduction to the symposium. Hortscience 25,52

Jeune B, Sattler R (1992) Multivariate analysis in process morphology of plants. J Theor Biol 156, 147167

Kervella J (1991) Variabilité génétique des caractéristiques morphologiques chez le pêcher (Prunus persica L Batsch). In : L'arbre, biologie et développement (C Edelin, ed). Nat Monsp hors série, 620-621

Lauri PE (1991) Données sur l'évolution de la ramification et de la floraison du pêcher (Prunus persica $\mathrm{L}$ Batsch) au cours de sa croissance. Ann Sci Nat Bot $13^{e}$ sér, t 11, 95-103

Lebart L, Morineau A, Warwick KM (1984) Multivariate descriptive statistical analysis. Correspondence analysis and related techniques for large matrices. John Wiley \& Sons, New York, $231 \mathrm{p}$

Lespinasse JM, Delort F (1988) Verger piéton. Fruits Légumes 50, 16-19

Lespinasse JM, Delort F (1990) Un arbre nouveau : obtention de cultivars nécessitant peu de taille et contrôlant naturellement leur mise à fruit. Arboric Fruit 433, 20-24

Monet R (1983) Le pêcher, génétique et physiologie INRA, Actualités scientifiques et agronomiques 12 . Masson, Paris, $134 \mathrm{p}$

Quinlan JD, Tobutt KR (1990) Manipulating fruit tree structure chemically and genetically for improved performance. Hortscience 25, 60-64

Rageau R (1982) Étude expérimentale des lois d'action de la température sur la croissance des bourgeons floraux du pêcher (Prunus persica L Batsch) pendant la post-dormance. CR Acad Agric Fr 68, 709-718

Scorza R (1984) Characterization of four distinct peach tree growth types. J Amer Soc Hort Sci 109, 455-457

Scorza R (1987) Identification and analysis of spur growth in peach (Prunus persica L Batsch). $J$ Hort Sci $62,449-455$

Scorza R, Lightner GW, Liverani A (1989) The Pillar peach tree and growth habit analysis of Compact $X$ Pillar progeny. J Amer Soc Hort Sci 114, 991-995

Siegel S (1956) Non-parametric statistics for the behavioral sciences. Series in psychology, McGraw-Hill, New York, $312 p$

White $\mathrm{J}$ (1979) The plant as a metapopulation. Ann Rev Ecol Syst 10, 109-145 